\title{
Studies on Clustering based on Minimal Ratio Spanning Tree in a Fuzzy Graph
}

\author{
S. Gountia \\ G. M.(Auto) College , Sambalpur \\ Orissa , India
}

\author{
S. K. Sahoo \\ Institute of Mathematics \& Applications, \\ Bhubaneswar-3, Orissa, India
}

\begin{abstract}
In this paper, A study has been made for Cluster Analysis using Minimal Ratio Spanning tree of a Fuzzy Graph. Different clusters are generated by deleting the edge(s) in consideration to specific property. Comparison has been studied with regard to Narrow Slicing procedure in the Fuzzy Graph.
\end{abstract}

General Terms: Fuzzy graph, Spanning tree, minimum ratio spanning tree in a fuzzy graph.

Key words: Min-max weight of an edge , min-max ratio edge connectivity

AMS Classificationnumber: (2000)-68T27/W31352

\section{INTRODUCTION}

Clustering is a process of grouping a set $X$ of objects into classes or clusters based on similarity. Graph-theoretic clustering methods are normally based on some kind of connectivity of the nodes of a graph representing the data set. The fuzzy graph approach is more powerful in cluster analysis than the usual graph theoretic approach. One of the best known graph-based divisive clustering procedure is based on the construction of the minimal spanning tree(MST) of the objects $[2,5]$. By the elimination of any edge from the MST we get sub trees which correspond to clusters. The concept of fuzzy graph with two weights were studied before [9], optimal ratio spanning tree in a fuzzy graph has been discussed[9]. Two types of weights, min-max weight of the cutset and min-max ratio weight of the cutsets [12] were introduced. Some works on clustering have been done by the authors applying min-max edge connectivity and min-max ratio edge connectivity with the application of Narrow Slicing Procedure. In this study Min-max ratio concept was also adopted before for determining the optimal ratio spanning tree in a fuzzy graph. An algebraic approach as well as a geometric approach has been used for the construction of minimal ratio spanning tree (MRST) using the min-max ratio concept and subclusters are obtained by the recursive division of clusters. Deletion of the edges from the spanning tree is based on the min-max-weights of the edges.

\section{PRELIMINARIES}

\section{Fuzzy graph:}

$G=(V, \sigma, \mu)$ is called a fuzzy graph if $\mathrm{V}$ is the non-empty set of vertices or nodes.

$\sigma: V \rightarrow[0,1]$, a fuzzy node set of $\mathrm{G}, \quad \mu: V \times V \rightarrow[0,1]$, a fuzzy edge set of $\mathrm{G}$ such that for all $x, y \in V, \mu(x, y) \leq \sigma(x) \wedge \sigma(y)$
Fuzzy sub graph:

$H=(P, \tau, v)$ is called a fuzzy subgraph of $G=(V, \sigma, \mu)$ if $P \subseteq V, \tau(x)=\sigma(x)$ and $v(x, y) \leq \mu(x, y)$ for all $x, y \in P$.

$\alpha$-cut fuzzy graph:

Let

$$
\sigma^{\alpha}=\{x \in V \mid \sigma(x) \geq \alpha\}
$$

$\mu^{\alpha}=\{(x, y) \in V \times V \mid \mu(x, y) \geq \alpha\} \quad$ for all $\quad \alpha \in[0,1]$. The sets $\mu^{\alpha}$ are called $\alpha$-cuts of $\mu$. Define a fuzzy graph

$G^{\alpha}=\left(V, \sigma^{\alpha}, \mu^{\alpha}\right)$ as $\alpha$-cut fuzzy graph if $\sigma(x) \geq \alpha$ and $\mu(x, y) \geq \alpha$ for all $x, y \in V$.

$\alpha$-cut Fuzzy subgraph:

A fuzzy subgraph $H^{\alpha}=\left(P, \tau^{\alpha}, v^{\alpha}\right)$ of a fuzzy graph $G=(V, \sigma, \mu)$ is called a $\alpha$-cut Fuzzy subgraph of $\mathrm{G}$ if $P \subseteq V, \tau(x)=\sigma(x)$ and $v(x, y) \leq \mu(x, y)$ i.e $\tau(x)=\sigma(x) \geq \alpha$ and $\quad \alpha \leq v(x, y) \leq \mu(x, y)$ for all $x, y \in P$

In order to stay in line with terminology of traditional graph theory we shall use the following definition of a fuzzy graph. For further use let us define the fuzzy graph as $G\left(x_{i}, x_{j}\right)=\left\{\left[\left(x_{i} x_{j}\right), \mu_{G}\left(x_{i}, x_{j}\right)\right] \mid\left(x_{i}, x_{j}\right) \in V \times V\right\}$ and fuzzy subgraph of $G\left(x_{i}, x_{j}\right)$ as $H\left(x_{i}, x_{j}\right)$ if the node set of $H$ is a subset of node set of $G$ and $\mu_{H}\left(x_{i}, x_{j}\right) \leq \mu_{G}\left(x_{i}, x_{j}\right)$ for all $\left(x_{i}, x_{j}\right) \in V \times V$.

Example 1 (Fuzzy graph)

Let $V=\left\{x_{1}, x_{2}, x_{3}, x_{4}\right\}$, then a fuzzy graph is described as $G\left(x_{i}, x_{j}\right)=$

$\left\{\left[\left(x_{1}, x_{2}\right), .7\right],\left[\left(x_{1}, x_{3}\right), .6\right]\left[\left(x_{2}, x_{1}\right), .5\right],\left[\left(x_{4}, x_{1}\right), .9\right],\left[\left(x_{4}, x_{3}\right), .2\right],\left[\left(x_{4}, x_{4}\right), 1\right]\right\}$ Spanning subgraph of a graph :

$$
\begin{array}{r}
\text { A subgraph } H\left(x_{i}, x_{j}\right) \text { spans the graph } \\
G\left(x_{i}, x_{j}\right) \text { if node sets of } H\left(x_{i}, x_{j}\right) \text { and } G\left(x_{i}, x_{j}\right) \text { are }
\end{array}
$$
equal, i.e they differ only in their weights.

Example 2

$$
\begin{aligned}
& \text { Let } G\left(x_{i}, x_{j}\right) \text { be defined as in example 1.Then } \\
& H\left(x_{i}, x_{j}\right)=\left\{\left[\left(x_{1}, x_{2}\right), .6\right],\left[\left(x_{1}, x_{3}\right), .6\right],\left[\left(x_{4}, x_{1}\right), .5\right],\left[\left(x_{4}, x_{3}\right), .1\right],\right\} \\
& \text { is a spanning subgraph of } G\left(x_{i}, x_{j}\right) \text {. }
\end{aligned}
$$


Definition:

A path in a fuzzy graph $G\left(x_{i}, x_{j}\right)$ is a sequence of distinct nodes $x_{0}, x_{1}, \ldots . x_{n}$ such that for all $\left(x_{i}, x_{i+1}\right), \mu\left(x_{i}, x_{i+1}\right)>0$. The strength of the path is $\min \left\{\mu\left(x_{i}, x_{i+1}\right)\right\}, i=0,1, \ldots . ., n-1$. The length of a path $n(>0)$ is the number of nodes contained in the path. Each pair of nodes $\left(x_{i}, x_{i+1}\right)$ for which $\mu\left(x_{i}, x_{i+1}\right)>0$ is called an edge (arc) of the graph. A path is called a cycle if $x_{0}=x_{n}$ and $n \geq 3$.

\section{Connected nodes:}

Two nodes that are joined by a path are called connected nodes. A fuzzy graph is connected if $\mu(x, y)>0$ for all $x, y$. Connectedness is a transitive relation.

\section{Tree in a fuzzy graph:}

A fuzzy graph is a forest if it has no cycles. If a forest is connected, then it is called a tree. (Thus tree in a fuzzy graph is a connected acyclic graph).

\section{Spanning tree in a fuzzy graph:}

A tree $T\left(x_{i}, x_{j}\right)$ is said to be a spanning tree of a connected fuzzy graph $G\left(x_{i}, x_{j}\right)$ if $T\left(x_{i}, x_{j}\right)$ contains all nodes of $G\left(x_{i}, x_{j}\right)$. Spanning trees of fuzzy graph described in

Example-1 are

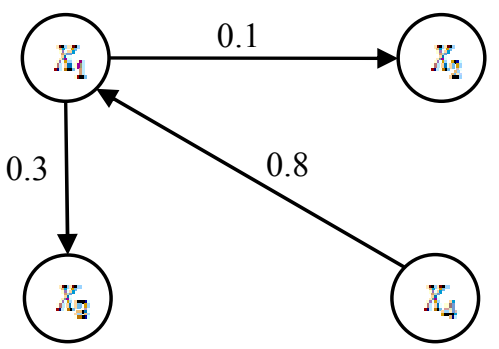

( Fig-1)

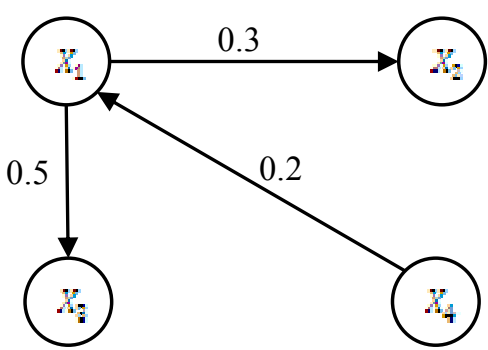

(Fig-2)

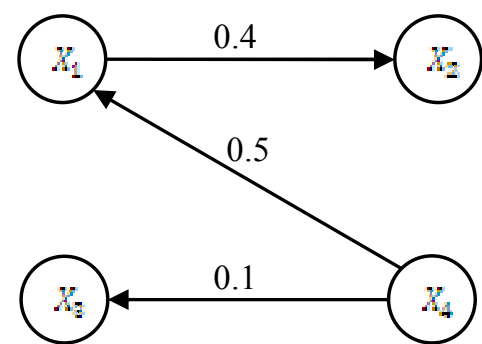

(Fig-3)

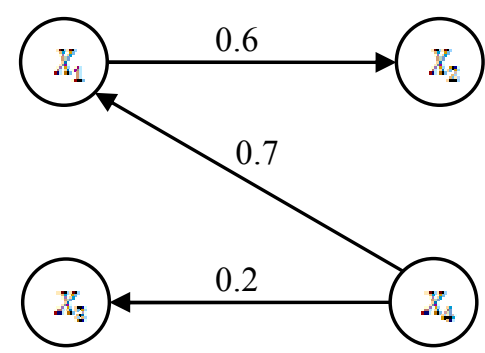

(Fig-4)

It is seen that for the same type of spanning tree (i.e for the same set of vertices and edges) there are infinite number of spanning trees..The spanning trees shown in Fig-1 and Fig-2 are of type- 1 and the spanning trees shown in Fig- 3 and Fig4 are of type- 2 .

\section{Definition:}

For a fuzzy graph with n-edges denoted by $e_{1}, e_{2}, e_{3} \ldots . e_{n}$ the spanning tree of type $\mathrm{i}$ (or spanning tree of category i) can be denoted by

$$
T^{i}=\left(e_{i_{1}}, e_{i_{2}}, e_{i_{3}} \cdots . . e_{i_{m}}\right) \text {,where the set }
$$

$\left\{e_{i_{1}}, e_{i_{2}}, e_{i_{3}} \ldots . . e_{i_{m}}\right\}$ with weights $\quad\left\{w_{i_{1}}, w_{i_{2}}, w_{i_{3}} \ldots \ldots w_{i_{m}}\right\}$ respectively is subset of the set $\left\{e_{1}, e_{2} \ldots \ldots e_{n}\right\}$.

$w_{i} \leq$ weights of $e_{i}, i=1,2 \ldots \ldots \ldots, k=1,2, \ldots, m, m \leq n$.

So, for a fuzzy graph with finite number of vertices and edges there are different categories of spanning trees and for each category of spanning tree there are infinite numbers of spanning trees with different weights.

\section{EDGE CONNECTIVITY \& NARROW SLICING}

Edge connectivity parameter, introduced by Yeh \& Bang [ 8] was used in fuzzy graph clustering. Studies have been made for fuzzy graph with each edge associated with two weights [9].

For a fuzzy graph $G=(V, \sigma, \mu)$ and for

$x, y \in V, \mu(x, y)=\left(\mu_{1}(x, y), \mu_{2}(x, y)\right) \quad$ such that $\mu_{1}(x, y) \leq \sigma(x) \wedge \sigma(y), \mu_{2}(x, y) \leq \sigma(x) \wedge \sigma(y)$. 
$\mu_{1}(x, y)$ is the $1^{\text {st }}$ weight of the edge $(x, y)$,

$\mu_{2}(x, y)$, is the $2^{\text {nd }}$ weight of the edge $(x, y)$

Min -max edge connectivity and Min-max ratio edge connectivity were introduced and narrow slicing procedure [ 4 ] is used for determining $\tau$-edge components.

\section{Definition:}

Let $G:(V, \sigma, \mu)$ be a fuzzy graph. Each edge $e$ of ${ }_{G}$ is associated with two weights $\mu_{1}(e)$ and $\mu_{2}(e) \cdot\left\{V_{1}, V_{2}\right\}$ be a partition of its vertex set $V$. The set of edges joining vertices of $V_{1}$ to $V_{2}$ is called a cut set of ${ }_{G}$ denoted by $\left(V_{1}, V_{2}\right)$ relative to the partition $\left\{V_{1}, V_{2}\right\}$. The weight of the cut set $\left(V_{1}, V_{2}\right)$ is defined to be $\sum_{u \in V_{1}, v \in V_{2}} \mu(u, v)$.

\section{Definition : (Edge Connectivity)}

Let ${ }_{G}$ be a fuzzy graph. The edge connectivity of $G$, denoted by $\lambda(G)$ is defined to be the minimum weight of the cut sets of $G_{G}, G$ is called $\tau$-edge connected if $G$ is connected and $\lambda(G) \geq \tau$. A $\tau$-edge component of $G$ is a maximal $\tau$-edge connected subgraph of ${ }_{G}$.

\section{Definition: Min-Max Ratio Weight (MMR-Weight)}

The min-max ratio weight (mmr-weight) of the cutset $\left(V_{1}, V_{2}\right)$ is defined to be

$$
\begin{aligned}
\mu^{R}(u, v)= & \frac{u \in V_{1}, v \in V_{2}}{\sum_{u \in V_{1}, v \in V_{2}}\left[\wedge\left(\mu_{1}(u, v), \mu_{2}(u, v)\right)\right]} \\
& =\frac{\left.\left.\sum_{e}\left[\wedge\left(\mu_{1}(e), \mu_{2}(e)\right)\right], \mu_{2}(u, v)\right)\right]}{\sum_{e}\left[\vee\left(\mu_{1}(e), \mu_{2}(e)\right)\right]}=\frac{\sum_{e}\left(\mu_{1} \cap \mu_{2}\right)(e)}{\sum_{e}\left(\mu_{1} \cup \mu_{2}\right)(e)}
\end{aligned}
$$

$e$ is the edge joining the vertices $u$ and $v, \mu_{1}(e)$ is the 1 st weight of the edge $e, \mu_{2}(e)$ is the 2 nd weight of the edge $e$. ' $\wedge$ ' stands for infimum, ' $\vee$ ' stands for supremum

\section{Definition : (MMR-Edge Connectivity)}

For a fuzzy graph ${ }_{G}$, the MMR-edge connectivity (min-max ratio edge connectivity) of ${ }_{G}$ denoted by $\lambda^{R}(G)$ is defined to be the minimum weight of mmr-weight of the cut sets of $G . G$ is called $\tau$-edge connected if $G$ is connected and $\lambda^{R}(G) \geq \tau$.A $\tau$ - edge component of $G$ is maximal $\tau$-edge connected subgraph of $G$.

\section{Definition : (MMR-Cohesiveness)}

Let $e$ be an element of a fuzzy graph $G$. The mmr-cohesiveness of $e$, denoted by $h(e)$ is the maximum value of mmr-edge connectivity of the subgraphs of $G$ containing $e$. Without loss of generality we can use the symbol $\lambda(G)$ instead of $\lambda^{R}(G)$.

\section{Example 3}

Consider the simple, connected undirected $\alpha$-cut fuzzy graph in Fig.5 with vertex set $V\left\{x_{1}, x_{2}, x_{3}, x_{4}, x_{5}, x_{6}\right\}$ and with $\quad \alpha=.1$. We have each $e_{j}$ corresponds to a pair $\left(\mu_{1} e_{j}, \mu_{2} e_{j}\right), j=1,2, \ldots \ldots \ldots \ldots \ldots . . .7$ i.e, $e_{1}(.3, .8), e_{2}(.4, .2)$, $e_{3}(.7, .6), e_{4}(.9, .7), e_{5}(.5, .1), e_{6}(.2,1)$ and $e_{7}(.6, .5)$.

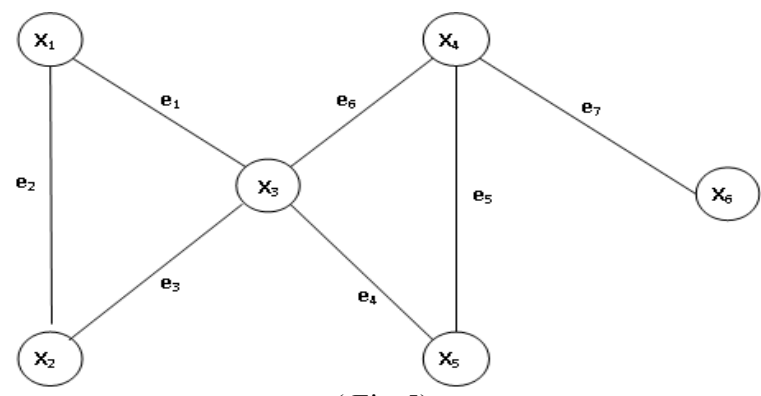

( Fig-5)

$\mu_{T}\left(x_{i}, x_{j}\right)=\mu_{G}\left(x_{i}, x_{j}\right)$ for all $\left(x_{i}, x_{j}\right) \in V \times V \cdot M_{\mu}$ is the corresponding fuzzy matrix of $G$ where $\left(M_{\mu}\right)_{i j}=\left(\mu_{1}\left(v_{i}, v_{j}\right), \mu_{2}\left(v_{i}, v_{j}\right)\right), v_{i}, v_{j}$ are the vertices of the graph.

$\begin{array}{lllllll} & \mathrm{x}_{2} & (0.4,0.2) & (0,0) & (0.7,0.6) & (0,0) & (0,0)\end{array}$

$M_{\mu}=\mathrm{x}_{3} \quad(0.3,0.8) \quad(0.7,0.6) \quad(0,0) \quad(0.2,1.0)(0.9,0.7)$ $(0,0)$

$\begin{array}{rrrrrrrr} & \mathrm{x}_{4} & (0,0) & (0,0) & (0.2,1.0) & (0,0) & (0.5,0.1) \\ (0.6,0.5) & & & & & \\ (0,0) & \mathrm{x}_{5} & (0,0) & (0,0) & (0.9,0.7) & (0.5,0.1) & (0,0) \\ & & & & & & \\ & \mathrm{x}_{6} & (0,0) & (0,0) & (0,0) & (0.6,0.5) & (0,0)\end{array}$

Slicing Procedure :-

Applying the Narrow Slicing Procedure [12], minmax ratio edge connectivity for subgraphs of $G$ and cohesiveness of edges are obtained.

The cohesive matrix $\mathrm{H}$ of the graph $\mathrm{G}$ is

$\begin{array}{lccccr}\mathrm{x}_{6} & \mathrm{x}_{1} & \mathrm{x}_{2} & \mathrm{x}_{3} & \mathrm{x}_{4} & \mathrm{x}_{5} \\ \begin{array}{c}\mathrm{x}_{1} \\ 0.000\end{array} \mathrm{~N}_{2} & 000 & 0.417 & 0.417 & 0.000 & 0.000 \\ \begin{array}{c}0.000 \\ \mathrm{H}=\mathrm{x}_{3}\end{array} & 0.417 & 0.857 & 0.000 & 0.381 & 0.778 \\ \begin{array}{c}0.000 \\ \mathrm{x}_{4}\end{array} & 0.000 & 0.000 & 0.381 & 0.000 & 0.381 \\ 0.381 & & & & & \end{array}$




$\begin{array}{cccccc}\mathrm{x}_{5} & 0.000 & 0.000 & 0.778 & 0.381 & 0.000 \\ \begin{array}{c}0.000 \\ \mathrm{x}_{6}\end{array} & 0000 & 0.000 & 0.000 & 0.381 & 0.000 \\ 0.000 & & & & & \end{array}$

and the narrow slicing of $\mathrm{G}$ is $\left(\left\{\mathrm{x}_{4}\right\},\left\{\mathrm{x}_{6}\right\},\left\{\mathrm{x}_{1}, \mathrm{x}_{2}, \mathrm{x}_{3}, \mathrm{x}_{5}\right\}\right)$, $\left(\left\{\mathrm{x}_{1}\right\},\left\{\mathrm{x}_{2}, \mathrm{x}_{3}, \mathrm{x}_{5}\right\}\right),\left(\left\{\mathrm{x}_{5}\right\},\left\{\mathrm{x}_{2}, \mathrm{x}_{3}\right\}\right),\left(\left\{\mathrm{x}_{2}\right\},\left\{\mathrm{x}_{3}\right\}\right)$. From this cohesive matrix $\mathrm{H}$ of $\mathrm{M}_{\mu}, \tau$-threshold graph of $\mathrm{H}$ can be obtained. Each component of the graph is a maximal $\tau$ edge connected graph.

The $\tau$-edge components of the graph $\mathrm{G}$ (example3,Fig-5 ) for various values of $\tau$ can be summarized as follows.

\section{Table-A}

$\tau$

$\tau$-edge components

$(0.857, \infty)$

$\left\langle\left\{x_{6}\right\}\right\rangle,\left\langle\left\{x_{4}\right\}\right\rangle,\left\langle\left\{x_{1}\right\}\right\rangle,\left\langle\left\{x_{5}\right\}\right\rangle,\left\langle\left\{x_{2}\right\}\right\rangle,\left\langle\left\{x_{3}\right\}\right\rangle$

$(0.778,0.857]$

$\left\langle\left\{x_{6}\right\}\right\rangle,\left\langle\left\{x_{4}\right\}\right\rangle,\left\langle\left\{x_{1}\right\}\right\rangle,\left\langle\left\{x_{5}\right\}\right\rangle,\left\langle\left\{x_{2}, x_{3}\right\}\right\rangle$

$(0.417,0.778] \quad\left\langle\left\{x_{6}\right\}\right\rangle,\left\langle\left\{x_{4}\right\}\right\rangle,\left\langle\left\{x_{1}\right\}\right\rangle,\left\langle\left\{x_{2}, x_{3}, x_{5}\right\}\right\rangle$

$(0.381,0.417] \quad\left\langle\left\{x_{6}\right\}\right\rangle,\left\langle\left\{x_{4}\right\}\right\rangle,\left\langle\left\{x_{1}, x_{2}, x_{3}, x_{5}\right\}\right\rangle$

$[0.0,0.381]$

$$
\left\langle\left\{x_{1}, x_{2}, x_{3}, x_{4}, x_{5}, x_{6}\right\}\right\rangle
$$

\section{MINIMAL RATIO SPANNING TREE}

For a connected $\alpha$-cut fuzzy graph $G^{\alpha}=\left(V, \sigma^{\alpha}, \mu^{\alpha}\right)$, with each edge associated with two weights, the min-max ratio concept has been applied for finding the optimal ratio spanning tree.

Let $G\left(x_{i}, x_{j}\right)$ be a connected $\alpha$-cut fuzzy graph with node set $V$, fuzzy node set $\sigma$, and fuzzy edge set $\mu_{G}$, where $\sigma: V \rightarrow[0,1], \sigma\left(x_{i}\right) \geq \alpha$

$$
\mu_{G}: V \times V \rightarrow[0,1], \mu_{G}\left(x_{i}, x_{j}\right) \geq \alpha
$$

,$\mu_{T}\left(x_{i}, x_{j}\right)=\mu_{G}\left(x_{i}, x_{j}\right)$ for all $x_{i}, x_{j} \in V, 0<\alpha \leq 1$

Associated with each edge $e_{i j}=\left(x_{i}, x_{j}\right)$ there are positive numbers (degrees of membership) $\mu_{1}\left\{e_{i j}\right\}$ and $\mu_{2}\left\{e_{i j}\right\}$, written as $\mu_{1} e$ and $\mu_{2} e$ respectively for notational convenience. So $\mu_{1} e$ and $\mu_{2} e$ are fuzzy weights of the edge e .

\section{Definition : (Min-max weight of an edge)}

The min-max weight of an edge ' $e$ ' is defined to be

$$
\mu^{*}(e)=\frac{\wedge\left(\mu_{1}(e), \mu_{2}(e)\right)}{\vee\left(\mu_{1}(e), \mu_{2}(e)\right)} .
$$

Definition : (Min-max ratio of spanning tree in a fuzzy graph)

Let $T\left(x_{i}, x_{j}\right)$ be any spanning tree of $G\left(x_{i}, x_{j}\right)$. Minmax ratio of $T\left(x_{i}, x_{j}\right)$ is denoted by

$$
\mu^{R}(T)=\frac{\mu_{1}^{R}(T)}{\mu_{2}^{R}(T)}=\frac{\sum_{e \in T} \wedge\left(\mu_{1}(e), \mu_{2}(e)\right)}{\sum_{e \in T} \vee\left(\mu_{1}(e), \mu_{2}(e)\right)}
$$

where $T=T\left(x_{i}, x_{j}\right)$, ' $\wedge$ ' stands for infimum and ' $\vee$ ' stands for supremum.

The problem of optimal ratio spanning tree with min-max ratio is concerned with determining a spanning tree $T\left(x_{i}, x_{j}\right)$ which optimizes the ratio $\mu^{R}(T)$ over the set of all spanning trees of $G\left(x_{i}, x_{j}\right)$.

\section{Objective Space:}

Through out, we discuss some special cases of trees in $\alpha$-cut fuzzy graph $G^{\alpha}=\left(V, \sigma^{\alpha}, \mu^{\alpha}\right)$ where $V$ is finite, and $\mu$ is symmetric. $\sigma(x) \geq \alpha$ and $\mu(x, y) \geq \alpha$ for all $x, y \in V, \mu_{T}\left(x_{i}, x_{j}\right)=\mu_{G}\left(x_{i}, x_{j}\right)$ for all $x_{i}, x_{j} \in V \times V$.

The optimal ratio spanning tree problem with minmax ratio $\mu^{R}(T)$ is to optimize the ratio $\mu^{R}(T)$ for all

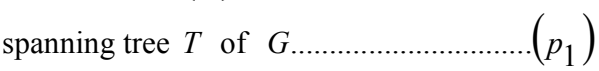

\section{Definition:}

The objective space in $R^{2}$ is defined as the convex hull $C$ of $\left(\mu_{1}^{R}(T), \mu_{2}^{R}(T)\right)$ where $T$ ranges over the set of all spanning trees of $G$. For each extreme point of $C$ there is a corresponding tree of $G$.

We illustrate the results by considering the following example given below.Consider the simple, connected undirected $\alpha$-cut fuzzy graph $\mathrm{G}$ of example-3

(Fig.5) with $\quad \alpha=.1$.

Then the possible types of spanning trees are as follows

$$
\begin{aligned}
& T^{1}=\left(e_{1}, e_{2}, e_{4}, e_{5}, e_{7}\right): T^{2}=\left(e_{1}, e_{2}, e_{5}, e_{6}, e_{7}\right): \\
& T^{3}=\left(e_{1}, e_{2}, e_{4}, e_{6}, e_{7}\right): T^{4}=\left(e_{2}, e_{3}, e_{4}, e_{5}, e_{7}\right): \\
& T^{5}=\left(e_{2}, e_{3}, e_{5}, e_{6}, e_{7}\right): T^{6}=\left(e_{2}, e_{3}, e_{4}, e_{6}, e_{7}\right): \\
& T^{7}=\left(e_{1}, e_{3}, e_{4}, e_{6}, e_{7}\right):: T^{8}=\left(e_{1}, e_{3}, e_{4}, e_{5}, e_{7}\right): \\
& T^{9}=\left(e_{1}, e_{3}, e_{5}, e_{6}, e_{7}\right)
\end{aligned}
$$


For each spanning tree $T^{i}, i=1,2,3, \ldots, 9$ the corresponding pair of weights are $\mu_{1}^{R}\left(T^{i}\right), \mu_{2}^{R}\left(T^{i}\right)$ where $\mu_{1}^{R}\left(T^{i}\right)=\sum_{e \in T} \wedge\left(\mu_{1}(e), \mu_{2}(e)\right)$ and $\quad \mu_{2}^{R}\left(T^{i}\right)=\sum_{e \in T} \vee\left(\mu_{1}(e), \mu_{2}(e)\right)$ The points $\left(\mu_{1}^{R}\left(T^{i}\right), \mu_{2}^{R}\left(T^{i}\right)\right)$ are plotted graphically in Fig6 in the objective space $R^{2}$.

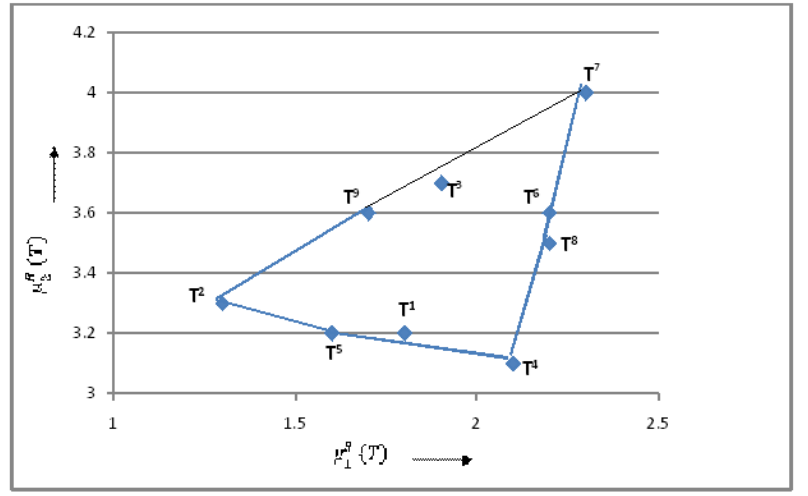

(Fig-6 : Convex hull of example-3)

From Fig-6 it is seen that the spanning tree $T^{2}$ has got the minimum value of $\mu_{1}^{R}(T)$ and $T^{7}$ has got the maximum value of $\mu_{2}^{R}(T)$.It can be easily verified that $T^{2}$ minimizes the ratio $\mu^{R}(T)$ and $T^{4}$ maximizes $\mu^{R}(T)$. Both $T^{2}$ and $T^{4}$ solve the problem and both of them are extreme points of $C$. From the above description it is observed that every spanning tree is not necessarily an extreme point of $C$, but every extreme point of $C$, corresponds to a spanning tree of $G$. Hence, $T^{2}=\left(e_{1}, e_{2}, e_{5}, e_{6}, e_{7}\right)$ is the Minimal ratio spanning tree of $\mathrm{G}$ with min-max ratio.

\section{Clustering Procedure:}

By the elimination of an edge from the MRST with minimum value of min-max weight we get subtrees which correspond to clusters. Clustering by minimal ratio spanning tree can be viewed as a hierarchical clustering procedure which follows the divisive approach.

Minimal ratio spanning tree of the graph $\mathrm{G}$ hasbeen formed with mmr weights. The MRST is denoted by $\mathrm{T}=\left(\mathrm{e}_{1}, \mathrm{e}_{2}, \mathrm{e}_{5}, \mathrm{e}_{6}, \mathrm{e}_{7}\right)$. The min-max weights of the edges are $0.357,0.5,0.2,0.2$ and 0.8333 for the edges $e_{1}, e_{2}, e_{5}, e_{6}$ and $\mathrm{e}_{7}$ respectively. Minimum weight of these weights is 0.2 corresponding to the edges $\mathrm{e}_{5}$ and $\mathrm{e}_{6}$. So deleting the edges $\mathrm{e}_{5}$ and $\mathrm{e}_{6}$ from $\mathrm{T}$ the subcluster obtained is $C_{1}=\left\{x_{4}, x_{6}\right\},\left\{x_{5}\right\},\left\{x_{1}, x_{2}, x_{3}\right\}$. Deleting $\mathrm{e}_{1}$, the edge with next higher weight i.e. 0.357 another subcluster $C_{2}=\left\{x_{4}, x_{6}\right\},\left\{x_{5}\right\},\left\{x_{1}, x_{2}\right\},\left\{x_{3}\right\}$ is obtained.

In this manner sub cluster $C_{3}=\left\{x_{4}, x_{6}\right\},\left\{x_{5}\right\},\left\{x_{1}\right\},\left\{x_{2}\right\},\left\{x_{3}\right\}$ $\&$ $C_{4}=\left\{x_{4}\right\},\left\{x_{6}\right\},\left\{x_{5}\right\},\left\{x_{1}\right\}, \quad\left\{x_{2}\right\},\left\{x_{3}\right\}$ can be obtained. We can summarize the $\tau$-edge components of $\mathrm{G}$ as follows.

Table-B

$(0.833, \infty)$

$(0.5,0.833]$

$\left\langle\left\{x_{4}\right\}\right\rangle,\left\langle\left\{x_{6}\right\}\right\rangle,\left\langle\left\{x_{5}\right\}\right\rangle,\left\langle\left\{x_{1}\right\}\right\rangle,\left\langle\left\{x_{2}\right\}\right\rangle,\left\langle\left\{x_{3}\right\}\right\rangle$

$(0.357,0.5]$

$\left\langle\left\{x_{4}, x_{6}\right\}\right\rangle,\left\langle\left\{x_{5}\right\}\right\rangle,\left\langle\left\{x_{1}\right\}\right\rangle,\left\langle\left\{x_{2}\right\}\right\rangle,\left\langle\left\{x_{3}\right\}\right\rangle$

$(0.2,0.357)$

$\left\langle\left\{x_{4}, x_{6}\right\}\right\rangle,\left\langle\left\{x_{5}\right\}\right\rangle,\left\langle\left\{x_{1}, x_{2}\right\}\right\rangle,\left\langle\left\{x_{3}\right\}\right\rangle$

$[0.0,0.2]$

$\left\langle\left\{x_{4}, x_{6}\right\}\right\rangle,\left\langle\left\{x_{5}\right\}\right\rangle,\left\langle\left\{x_{1}, x_{2}, x_{3}\right\}\right\rangle$

$[0.0,0.2] \quad\left\langle\left\{x_{1}, x_{2}, x_{3}, x_{4}, x_{5}, x_{6}\right\}\right\rangle$

\section{CONCLUSION}

Study has been made for generation of clusters by deletion of specific edge from MRST and by application of Narrow Slicing Procedure with mmr-weights in a fuzzy graph. It is observed with the described examples that the clusters obtained are different and by deletion of edges with minimum value of min-max weights from a minimal ratio spanning tree better clusters are obtained.

\section{ACKNOWLEDGEMENTS}

Our thanks to the experts who have contributed towards development of the template.

\section{REFERENCES}

[1] A. vathy-Fogarassy, B.Feil, J.Abonyi”Minimal Spanning Tree based Fuzzy clustering" Proceedings of World academy of Sc., Eng \& Technology, vol-8, Oct2005, 7-12.

[2] Barrios-Gonzales, J.M., Quiroz, A.J., "A clustering procedure based on comparision between the $\mathrm{k}$ nearest neighbours graph and the minimal spanning tree "In Statistics \& Probability Letter, 62: 23-34., 2003.

[3] C.T., Zahn, "Graph-theoretical methods for detecting and describing gestalt clusters " IEEE Transaction on Computers, C 20: 68-86., 1971.

[4] D.W.Matula, $k$-components, clusters and slicing in graphs, SIAM J. Appl. Math. 22: 459-480(1972).

[5] J.C. Bezdek, J.C, Gower, Ross G.J.S., "Minimal Spanning Trees and Single Linkage Cluster Analysis", Applied Statistics, Vol. 18, pp. 54-64., 1969.

[6] J.N.Mordeson and P.S.Nair, Fuzzy graphs and Fuzzy Hyper graphs, Physica Verlag, studies in Fuzziness and soft computing, 46(2000). 
[7] P.Swain and A.K.Pujari: A new Algorithm to minimum ratio spanning tree, Journal of Management system, Vol.13, No.2,(1997),197-206.

[8] R.T.Yeh,S.Y.Bang, Fuzzy relations , fuzzy graphs and their applications to clustering analysis , in:La.Zadeh,K.S.Fu.M.Shimura(Eds.),Fuzzy sets and Their Applications, Academic Press, 1975.pp.125-149.

[9] S.K. Sahoo and S. Gountia, "Optimal ratio spanning tree in $\alpha$-cut fuzzy graph; A Geometrical Approach " Ultra Scientist of Physical Sciences, Vol. 21(1)M, (2009) 5160 .
[10] S.Gountia and S.K.Sahoo, "Studies on Fuzzy Tree and Fuzzy Forest in a Fuzzy Graph" Journal. Of Orissa Mathematical Society, vol-28, (No1 \&2),(2009) 87-97.

[11] S.Gountia and S.K.Sahoo, "Studies on clustering, based on edge-connectivity in a Fuzzy graph", Ultrascientist of physical sciences, Vol-22(2)M, (2010).

[12] S. Gountia and S.K.Sahoo, "On Clustering, based on Edge-Connectivity in a Fuzzy Graph " Journal of Orissa Mathematical Society Vol-29 (No. 1\&2)(2010) 151172.

[13] S.Gountia and S.K.Sahoo, "Edge Connectivity based Clustering in a fuzzy graph " Proceedings of the International Conference MMIP-2011 , N.I.T. ,Calicut , India. 